\title{
Some correlates of inhibition-facilitation effect on reaction-time due to unexpected increase in stimulus intensity'
}

\author{
ARJUH P. PUROHIT \\ QUEEN'S UNIVERSITY, KINSTON, UNTARIO
}

Ss who showed an inhibitory effect in reacting to an auditory stimulus, the intensitv of which was increased unexpectedly, were compared with $S$ s who showed a facilitatory effect in reacting to a similar stimulus. No difference was noticed between the two groups in introversion, anxietyneuroticism and autonomic lability measures. There was a significant negative correlation between latency of reaction to a weak stimulus and inhibition-facilitation effect. This result is discussed in terms of the curvilinear performance theory of activation and an alternative explanation is offered.

It is a common observation that some people "freeze" and some react quite violently when they suddenly come across an emergency situation. The purpose of this study was to investigate possible correlates to this inhibition-facilitation phenomenon in a reaction-time type situation.

Introversion tests were used to find out whether inhibitor and facilitators can be discriminated. The rationale for using introversion as a variable was Eysenck's statement: "We are led to postulate two extreme types of reaction, depending upon presence or absence of strong inhibitory potential. The strongly inhibited type (extrovert; hysteric) should on this hypotheses be subject to sluggish, inert response behaviour; the weakly inhibitory type (introvert; dysthymic) should be subject to over-active over-responsive behaviour" (Eysenck, 1957, p. 135).

Anxiety-neuroticism tests were used for the same purpose. Wenar (1954) reports that anxious Ss reacted faster than non-anxious Ss to weak but not to strong stimuli; these results support Spence's chronic theory of anxiety (Spence, 1956). Castaneda (1956), however, found that reaction speed increases with stimulus intensity much more in anxious than in non-anxious Ss; this is a finding consistent with Spence's reactive theory of anxiety (Spence, 1956). Therefore, there would appear to be no explanation for the behavior of inhibitors in Spence's theoretical framework regardless of the possible validity of either theory.

Two autonomic lability measures were used in this study to verify an extension of Sternbach's (1960) finding that slow reactors to the sound of a pistol shot showed greater changes in several autonomic indices than did fast reactors. Two procedural changes were made. First, Ss received a strong stimulus when expecting a weak stimulus while in Sternbach's study, Ss were aware that the stimulus would be strong. Secondly, autonomic lability scores were obtained prior to the reaction- time experiment whereas Sternbach recorded autonomic changes during the reaction-time experiment. Besides, the question was examined whether sex-difference and response speed to a weak stimulus are in any way related to inhibition-facilitation phenomenon.

Method

Subjects. Sixty-four male and 64 female undergraduate students volunteered for this experiment.

Procedure. The main experiment as well as other objective testing and autonomic index recording was conducted in a soundproof room. Prior to the main experiment scores on the different individual difference variables were obtained. Introversion scores were obtained from the MPI-E Scale (Eysenck, 1957), the Kinaesthetic After-effect Test (Eysenck, 1955), the Serial Reactiontime Test (Eysenck \& Claridge, 1962), resistance to GSR adaptation (Eysenck, 1963) and frequency of spontaneous GSRs. ${ }^{2}$ Anxiety-neuroticism scores were obtained from the MAS (Taylor, 1953) and MPI-N scale (Eysenck, 1959). GSR and heart-rate lability scores were obtained from the GSR and the heart-rate records of the Ss. Hearing level of Ss' ears was estimated by an Amplirox audiometer. Details of administration and scoring of the foregoing tests are reported elsewhere (Purohit, 1966).

After the above tests were completed, the Ss were instructed to press a reaction-time key as soon as they heard auditory stimuli. Pure tones of $500 \mathrm{cps}$ and $\mathbf{0 . 5}$ sec. duration were presented to the S's better ear through an earphone from a sound generator at intervals varying from 30 to $40 \mathrm{sec}$. The first three stimuli were at $70 \mathrm{~dB}$ and the fourth at $120 \mathrm{~dB}$ above hearing level of the S's better ear. Latency of reaction in each case was recorded by a timer. Ss were not advised of any change in intensity of stimulus and, following the experiment, were requested not to discuss the experimental procedure with others.

Resulis

The inhibition-facilitation (I-F) scores were obtained for each $\mathrm{S}$ by deducting his latency of reaction to the third weak stimulus $\left(\mathrm{RT}_{\mathrm{w}}\right)$ from his latency of reaction to the strong stimulus $\left(\mathrm{RT}_{\mathrm{S}}\right)$. The distributions of inhibitors $\left(\mathrm{RT}_{\mathrm{S}}-\mathrm{RT}_{\mathrm{W}}=+\right)$ and facilitators $\left(R \mathrm{~T}_{\mathrm{S}}-\mathrm{RT}_{\mathrm{W}}=-\right)$ were examined for possible sex-differences. It was found that among the males there were 19 inhibitors and 39 facilitators and among the females there were 20 inhibitors and 34 facilitators. Six males and 10 females had zero I-F score. Therefore sex-difference does not seem too important in this distribution. 
Then it was examined whether inhibitors and facilitators differed in any personality variables. Twenty extreme inhibitors and 20 extreme facilitators, each group consisting of equal number of males and females, were selected from the total sample for this purpose. Then a 2 (sexes) by 2 (I-F) analysis of variance was performed on their scores on all the 10 personality measures. None of the F-ratios for the main effect of I-F and those for the interaction between sex and I-F was significant at .05 level.

Next, the relation between $\mathrm{RT}_{\mathrm{W}}$ and I-F scores was examined. It was found that out of $20 \mathrm{Ss}$ with shortest $\mathrm{RT}_{\mathrm{W}}$ (that is, the speediest reactors), 18 had inhibitory and two facilitatory scores. Conversely, out of $20 \mathrm{Ss}$ with longest $\mathrm{RT}_{\mathrm{W}}$ (that is, the slowest reactors), four had inhibitory, 15 facilitatory and one zero I-F scores. The tetrachoric correlation between $\mathbf{R T}_{\mathrm{W}}$ and I-F scores from this distribution is -0.89 . When the Pearson's $r$ was computed between $\mathrm{RT}_{\mathrm{W}}$ and I-F scores of all $\mathrm{Ss}$, the correlation was reduced to -0.32 (significant at .01 level). Then on the basis of $\mathrm{RT}_{\mathrm{w}}$, the entire sample was divided into eight equal groups and mean I- F scores were obtained for each group. Figure 1 graphically represents the linear relationship between the two indices.

\section{Discussion}

From these results it is obvious that sex-difference and individual differences in terms of introversion, anxiety-neuroticism and autonomic lability had no relationship with the I- F phenomenon. It is difficult to relate these results to the predictions derived from theories of Eysenck and Spence. On the other hand a discernible relationship was seen between the response speed to the weak stimulus and the I-F phenomenon

These results might possibly be interpreted in accordance with the now popular curvilinear performance theory of activation (Hebb, 1955; Malmo, 1959; Duffy, 1952). If $\mathrm{Ss}$ with longer $\mathrm{RT}_{\mathrm{W}}$ are assumed to be at the low-end of the activation continuum and those with shorter $\mathrm{RT}_{\mathrm{W}}$ at the middle portion, it could be argued, first, that a sudden increase in stimulus intensity will increase the activation level in both groups and, second, that the first group consequently would perform like

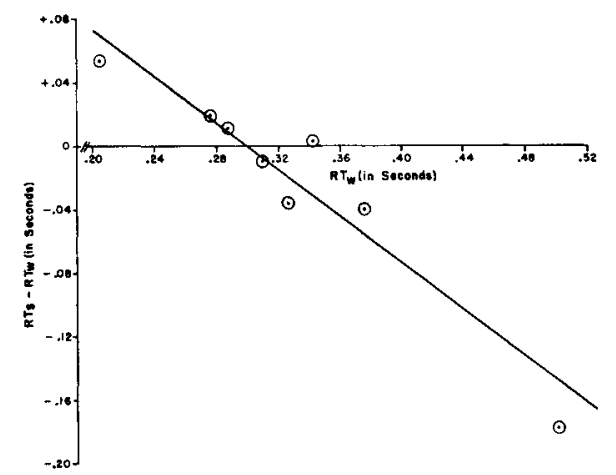

Fig. 1. Relationship between latency of reaction to third weak $\left(R T_{w}\right)$ and the difference between $R T_{w}$ and latency of reaction to the strong stimulus $\left(\mathbf{R T}_{\mathbf{S}}\right)$. persons at the middle of the continuum, and the second group like persons at the high end. The negative correlation observed between $R T_{W}$ and $R T_{S}-R T_{W}$ would be consistent with these assumptions.

Three possible objections can be raised to this interpretation. First, the assumptions as to positions may be false since inhibitors and facilitators did not differ on the anxiety-neuroticism or autonomic lability measures. Sternbach's results, however, strongly suggest that greater autonomic changes can be observed in inhibitors if recordings are made during the reaction. The negative results of this study might be attributed to the instability of the autonomic lability measures and the lack of validity of the introversion and the anxiety-neuroticism measures as activation measures.

Secondly, the curvilinear performance theory of activation would lead to the prediction that the correlation between $\mathrm{RT}_{\mathrm{W}}$ and $\mathrm{RT}_{\mathrm{S}}$ would be negative. In fact, it was +0.225 .

Finally, the curvilinear performance theory of activation does not explain why Ss giving $\mathrm{RT}_{\mathrm{w}}$ or medium latency did not change their latency of reaction to the strong stimulus (see Fig. 1).

A more likely explanation seems to be that the sudden increase in the stimulus had relatively stronger inhibitory than facilitatory effect in the case of persons with short $\mathrm{RT}_{\mathrm{W}}$, relatively stronger facilitatory than inhibitory effect in the case of persons with long $\mathrm{RT}_{\mathrm{w}}$, and inhibitory and facilitatory effects of equal strength in the case of people with $\mathbf{R T}_{\mathbf{W}}$ of medium latency.

\section{References}

Castanedo, $\boldsymbol{A}$. Reaction time and response amplitude as a function of anxiety and stimulus intensity. J. abnorm. soc. Psychol., $1956,53,225-228$.

Duffy, E. Activation and behaviour. New York: John Wiley \& Sons, 1962.

Eysenck, H. J. Cortical inhibition, figural after-effect and theory of personality. J. abnorm. soc. Psychol., 1955, 51, 94-106.

Eysenck, H. J. The dynamics of anxiety and hysteria. London: Routledge \& Kegan Paul, 1957.

Eysenck, H. J. Manual of Maudsley personality inventory. London: University of London Press, 1959.

Eysenck, H. J. Experiments with drugs (Ed.), London: Pergamon Press, 1963.

Eysenck, H. J., \& Claridge, G. S. The position of hysterics and dysthymics in a two dimensional framework of personality description. J. abnorm. soc. Psychol., 1962, 64, 46-55.

Hebb, D. O. Drives and the CNS. Psychol. Rev., 1955, 62, 243-254.

Malmo, R. B. Activation: A neuropsychological dimension. Psychol. Rev., 1959, 66, 367-386.

Purohit, A. P. Personality variables, sex-difference, GSR responsiveness and GSR conditioning. J. exp. Res. Person., 1966, in press.

Sternbach, R. A. Correlates of differences in time to recover from startle. Psychosom. Med., 1960, 22, 143-148.

Taylor, J. A. A personality scale of manifest anxiety. J. abnorm. soc. Psychol., 1953, 48, 285-290.

Spence, K. W. Behavior theory and conditioning. New Haven: Yale University Press, 1956.

Wenar, $C$. Reaction time as a function of manifest anxiety and stimulus intensity. J. abnorm. soc. Psychol., 1954, 49, 335-340. Notes

1. This experiment was conducted in the University of Western Australia.

2. Personal communication. 